\title{
Optical Frequency Measurement: 40 Years of Technology Revolutions
}

\author{
John L. Hall
}

Invited Paper

$\mathbf{T}$ HE PAST forty years have witnessed spectacular progress in precision measurements, beginning with the first coherent optical source, the $\mathrm{HeNe} \mathrm{CW}$ laser, demonstrated in late 1960 by A. Javan, B. Bennett, and D. Herriott, at Bell Laboratories. Almost immediately, these pioneers of the stable laser epoch introduced optical heterodyne techniques to explore the stability of the laser's optical frequency, expecting the few milliHertz linewidth predicted by the Schawlow-Townes formula for phase diffusion, and instead finding myriad physical processes that broaden and jiggle optical frequencies far beyond that narrow range. After a huge time and learning effort, most of these technical limitations can now be overcome. Considering that the central frequency is some $5 \times 10^{14} \mathrm{~Hz}$, comparison with a potential resolution of these few millihertz reveals a potential dynamic range of $10^{17}$ and generates an excitement which is palpable, perhaps increasingly so even today, almost forty years later. In plain language, this untapped dynamic range of resolution offers one of our best tools for discovering new physics, in just the next decimal.

This paper is intended to offer a bystander/sometimes- participant's view into the advances in laser tools and the exciting scientific/technical worlds that were opened by them. Roughly one "zillion" further clever inventions and techniques have added capability to the laser and have enabled many bursts of productive research. Now, after nearly forty years of incremental progress, the astonishing reality is that-within the past one year-absolute optical frequency measurements have suddenly become feasible, simple - even routine. This welcome and unexpected progress is based on the merger of techniques from the ultrastable lasers and the ultrafast lasers research communities. In a very real sense, these research objectives seem to be the exact opposites - the most unchanging versus the most quickly changing. But let's begin the story by listing a few selections out of the one zillion definite advances, starting with the $\mathrm{CW}$ gas laser itself:

1) $\mathrm{CW} \mathrm{HeNe}$ gas laser 1960;

2) Optical heterodyne diagnostic of frequency behavior 1961;

3) Proposals for measurement of the speed of light 1961 ;

4) Frequency-based control of laser frequency 1968;

Manuscript received November 16, 2000.

The author is with JILA, the National Institute of Standards and Technology, and the University of Colorado, Boulder, CO 80309-0440 USA.

Publisher Item Identifier S 1077-260X(00)11601-6.
5) Optical frequency stabilization with nonlinear spectroscopy 1966;

6) Optical frequency measurement with harmonic generation chains;

7) Speed of light experiment using beat frequencies and long-path interferometry;

8) Rapid progress in harmonic frequency measurement—chains reach $10.6 \mu \mathrm{m}$;

9) Stopped long-path interferometry for measuring $c$ : start direct $\lambda$ measurement 1970;

10) Frequency-controlled interferometry;

11) Speed of light via $\lambda * \nu=c[1]$;

12) Confirmation by NPL, NRC, PTB, and NRLM;

13) Redefinition of the Meter (1983);

14) Tunable dye lasers;

15) Frequency control of tunable dye lasers (1973);

16) Tunable and frequency-controlled solid-state lasers;

17) Spectroscopy with frequency-controlled tunable lasers 1978;

18) Resolution enhancement with larger laser beams Ramsey fringes (1975-1977);

19) Laser-based atom and ion cooling suggestions (1975);

20) Using time-periodic pulses to create frequency-periodic comb (1978);

21) Frequency synthesis using multiples of difference frequencies at $10 \mu \mathrm{m}$;

22) Frequency synthesis using frequency interval-divider stages $(1990+)$;

23) Revolution in resolution with cold atoms and stable lasers $(1998+)$;

24) Femtosec laser arrives - comb bandwidth is 5\% and up (1992);

25) First direct optical frequency measurements, combining divider stages and comb (1999);

26) Comb bandwidth extension using nonlinear fiber response $30 \%$ BW (2000);

27) Microstructure fiber with controlled GVD allows octave comb generation (2000);

28) Direct one-step microwave to visible frequency synthesis (2000);

29) Optical frequency stability transferred to comb (2000);

30) FS pulse synchronization demonstrated (2000).

Surely this is an incomplete list of relevant and important topics. But, even for these, in the allotted time and space there could be no full treatment given for everything. Therefore, we will focus 
on the issue of "Optical Frequency Measurement: Then and Now."

One of the first dreams brought by the CW laser was the prospect to measure the speed of light. Of course, the frequency of near-visible light is far too high to measure directly-until last year-but difference frequency schemes abounded. Soon after the original 1152.590-nm Ne line was found, other transitions in the same multiplet were discovered also to be capable of laser action. Most of these were at completely different colors. The closest line would give beat frequencies of hundreds of gigahertz or more-far too much to measure by any methods known at the time. To extend the frequency range, one new idea was to rapidly scan an electron beam produced from such a high beat frequency. Suppose one were transversely scanning at a rate very near a subharmonic of the beat frequency: then a stroboscopic pattern would be formed in angle, yielding a slowly moving pattern of dots on the CRT phosphor. Looking at a single spot, or at all through a matching mask, would allow measurement of the slower difference frequency. Harold S. Boyne and Zoltan Bay joined Peter L. Bender and the writer in this project. A Contract was made with EG \& G to build such a special CRT, based on their traveling-wave microwave CRT design. Unfortunately, it soon became clear that the S1 (IR-sensitive) photocathode could not withstand anything like the designed $20-\mathrm{kV} / \mathrm{cm}$ extraction field without arcing, and the project was dropped. Eventually a new laser line just $51 \mathrm{GHz}$ away was identified at $1152.818 \mathrm{~nm}$ [2]. This was great news as one could dream of detecting this frequency directly with a Schottky diode. Indeed, this proved possible [3] by driving the diode's capacitive reactance in the reverse-bias regime with a strong applied microwave field.

The prospect of an eventual precise measurement of the difference in frequency between two lines was positively exhilarating when taken with the clear possibility of measuring the wavelength difference interferometrically. This will immediately give a new measurement of the speed of light:

$$
c=\frac{f_{1}-f_{2}}{\frac{1}{\lambda_{1}}-\frac{1}{\lambda_{2}}} .
$$

Obviously, optical frequencies are (only seem!) too high to ever be directly measured, but with this differential approach we surely would be able to get a measurement more accurate than the Froome [4], [62] microwave value! An abandoned gold mine was identified (the "Poorman's Relief Gold Mine" about 10 miles west from Boulder). Soon, under the leadership of Peter Bender, Jim Faller, and the author were joining him in this serious effort of constructing an isolated, evacuated 30-m high-finesse interferometer in a place that was fully moist and dirty. Fig. 1 shows the 30-m vacuum pipe and the piers and optics chambers. Barometric pressure was isolated from the piers by using bellows and equivalent apertures on each side of the box. This optical path was remarkably stable, much better than our Lamb-dip-stabilized red HeNe lasers of the day. But the laser system did offer reasonable long-term stability. So it was quite a surprise that at an inland location, $2000 \mathrm{~km}$ from the nearest ocean, we received and measured extremely good signals with

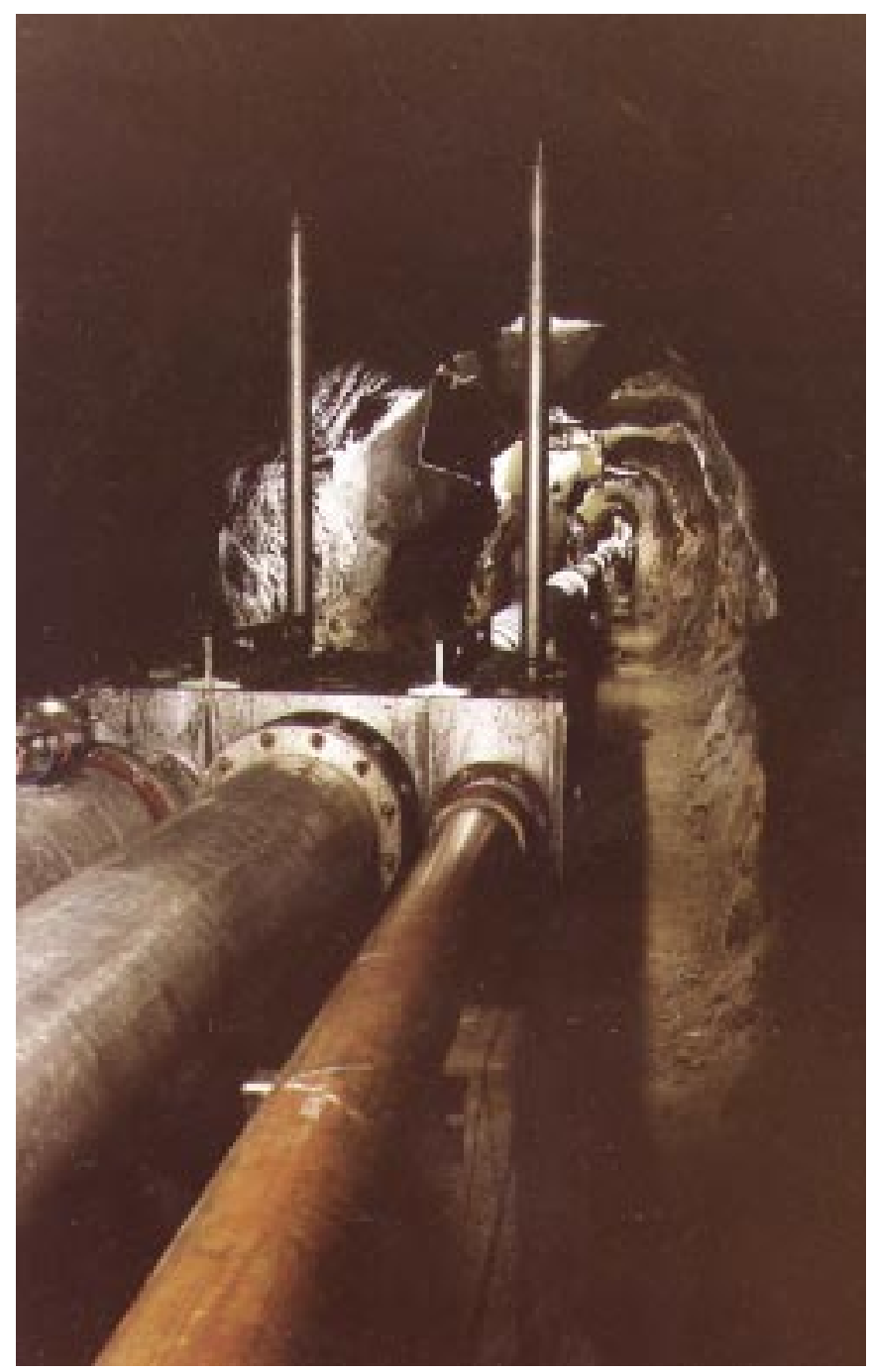

Fig. 1. View of 30-m interferometer in Poorman's Relief Gold Mine, with a colleague adjusting a mirror at the far end.

one and two cycles per solar day: earth tides in the rock crust of the earth! The peak-peak strain amplitudes were near $3 \times 10^{-8}$.

The earth was quite stable compared with our lasers, at least in the short term. What was needed was a narrower stronger reference line for the locking job. Paul Lee and Mike Skolnick had shown how to use separate intracavity gain and absorption cells [5]. With Dick Barger, we used the spectral overlap of the 3.39- $\mu \mathrm{m}$ HeNe line with a nice absorption line in $\mathrm{CH}_{4}$. Basically, this urgent need for laser stability led directly to the invention of the first of the lasers stabilized by saturated absorption of molecular transitions. The saturated absorption process gave very good signals with narrow lines and a surprising absence of shifts. Already, in 1969, a frequency stability of $10^{-13}$ and reproducibility of $10^{-11}$ was obtained [6]. Fig. 2 shows the Methane-stabilized reference laser working in the Poorman's Relief Mine, along with the $3.39-\mu \mathrm{m}$ tunable laser locked onto the Finesse $=44$ fringes in the long path interferometer. The beat frequency provides readout [7] of the interferometer length changes caused by earth tides, geophysics, and even underground nuclear tests. This methane-based laser stabilization system remains one of the best secondary frequency references, second only perhaps 


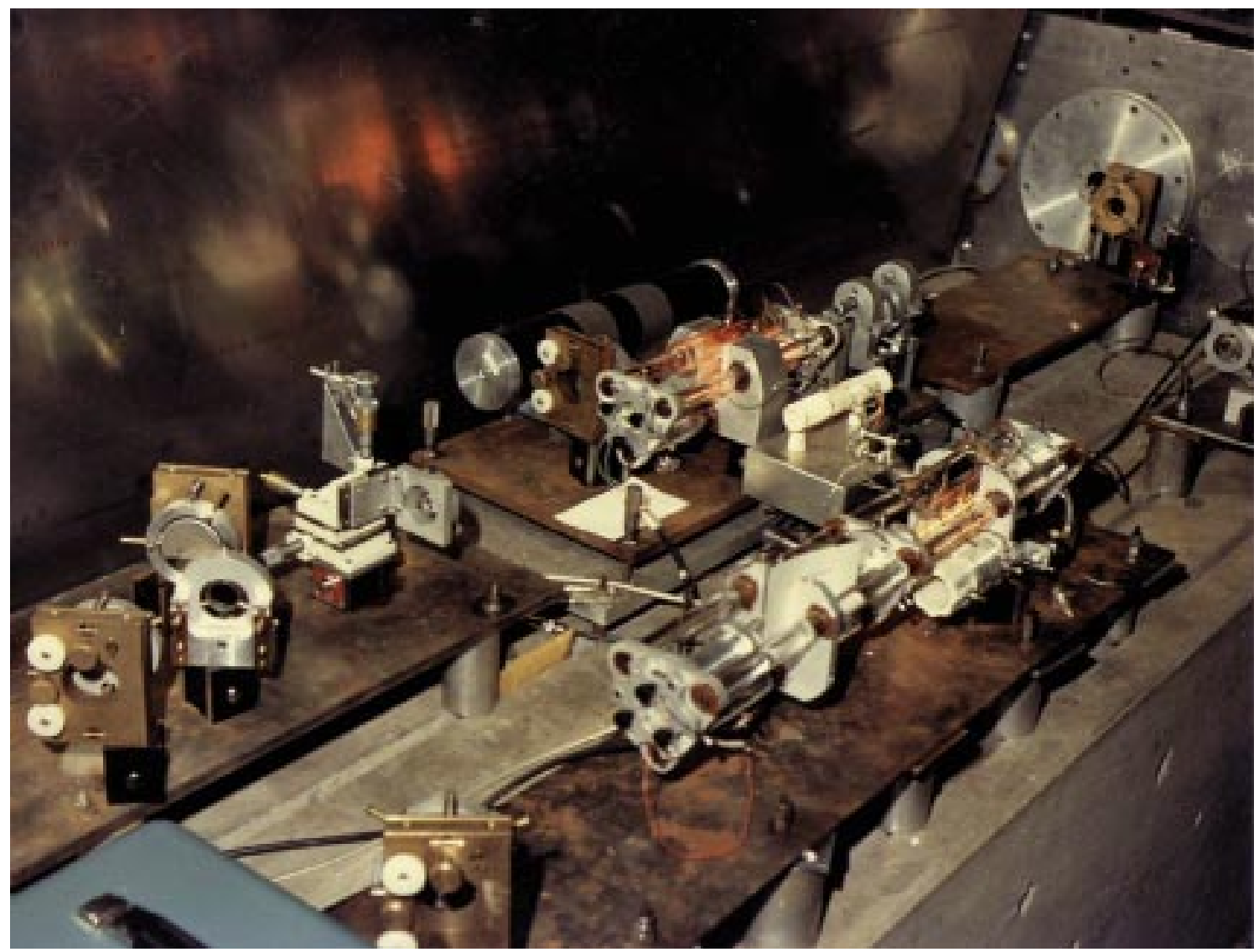

Fig. 2. Laser table, in next room, to left and behind camera position of Fig. 1. Methane-stabilized laser (longer laser in foreground) heterodynes with interferometer-cavity length-stabilized laser, to provide frequency-based length control or readout information $(\sim 1971)$.

to the analogous system at $10.6 \mu \mathrm{m}$ using $\mathrm{OsO}_{4}$ absorbers with a $\mathrm{CO}_{2}$ laser [8].

Interestingly, as so often happens in science, the same idea for a speed of light measurement was being studied elsewhere, namely by Veniamin Chebotayev [9] and his associates at the Institute for Semiconductor Physics, of the Academy of Sciences of the USSR, in Novosibirsk, Siberia. Chebotayev had even identified the same laser line pair and explored discharge conditions which would tend to favor the weaker line: this was to skip the helium and use a hydrogen-neon mixture. We had found that pure neon gave much less output power, but enough power to obtain heterodyne results for the $26-\mathrm{GHz}$ beat at 3.39 $\mu \mathrm{m}$.

Of course, the laser was the latest and coolest scientific thing, attracting much effort worldwide: Every potential laser system was being tried and soon many new transitions were shown to support laser action. Many lines were found in molecular discharges containing methyl alcohol, $\mathrm{D}_{2} \mathrm{O}$, water, $\mathrm{HCOOH}$, etc. Even $\mathrm{HCN}$ gave lasing action, at the surprisingly low frequency/long wavelength of $890 \mathrm{GHz} / 337 \mu \mathrm{m}$. Optically pumping some molecular laser gases with the output of a line-tunable $\mathrm{CO}_{2}$ laser led to just zillions of infrared transitions. Of interest in this story was the work by Ali Javan (who by then was Professor Javan of MIT) and his students who had begun using fast Point-Contact Silicon Diodes. Initially these units were microwave detectors with the cartridge modified so that a laser beam could be focused near the active surface region. Driving the diode with strong microwave radiation led to useful levels of nonlinear response. One of the first results of this genre was the measurement of the $\mathrm{HCN}$ laser at 890 $\mathrm{GHz}$ by Hocker, Javan, and co-workers [10]. Of course, this set off a cascade of next-frequency measurements, typically at a frequency twice as high as the last record. As the wavelength became shorter, it was found that silicon was becoming less efficient, and finally the now-standard metal-insulator-metal (MIM) tunneling junctions came to be the mixer of choice. Its bandwidth was truly impressive: the heterodyne beat frequency just didn't matter. Soon summing two lasers or, even easier, generating "optical" harmonic frequencies became popular. Now one didn't need exact submultiple relationships between the lasers-sums or differences could span the needed intervals. Three-lasers-plus-a-microwave approaches led to the possibility to synthesize many frequencies.

In the late 1960s, Ken Evenson, Joe Wells, and Don Jennings at NBS (now NIST) in Boulder came into this business in a big way. A corresponding team formed at the National Physical Laboratory (UK). After all, for a standards laboratory, what better thing is there to measure than the speed of light? For this, one would measure whatever laser gave the highest measurable frequency, and then set up an interferometer to measure its wavelength also. So even though a serious effort had been invested in the Poorman Mine 30-m interferometer, it became amply clear to us that the best way to get to the speed of light was to organize to help the direct frequency measurement team. 


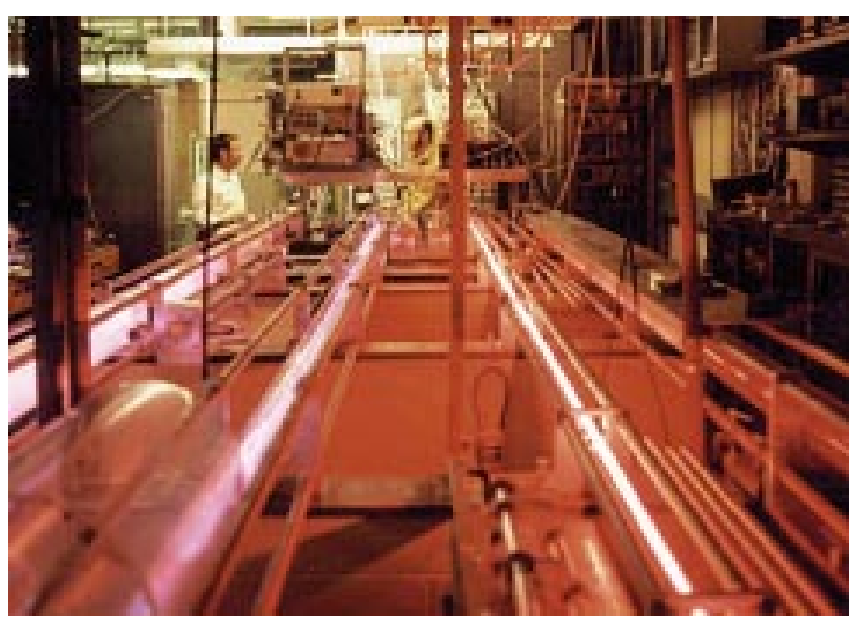

Fig. 3. Laser table in NIST labs for Frequency of Methane-stabilized Laser Experiment. Gain tubes are $8 \mathrm{~m}$ long to achieve adequate power for nonlinear mixing. J. S. Wells (left) and K. M. Evenson (center, back).

At this point, Judah Levine took over the 30-m interferometer for high-precision geophysical studies [7], but that is another story.

To have sufficient optical power for the NBS/NIST harmonic frequency chain measurement of the frequency of the methane transition at $3.39 \mu \mathrm{m}$, Evenson, Wells, and Jennings set up lasers $8 \mathrm{~m}$ long. Fig. 3 shows Evenson and Wells in a 1972 photo with these toy lasers, the basis of the NBS/NIST harmonic frequency chain. The leftmost is a discharge-based HCN laser emitting at $890 \mathrm{GHz}(337 \mu \mathrm{m})$. Next is a long $\mathrm{H}_{2} \mathrm{O}$ laser at $10.7 \mathrm{THz}$ (28 $\mu \mathrm{m})$, locked to the 12th harmonic of HCN [11], [63]. The next one is a powerful $\mathrm{CO}_{2}$ laser used to capture the synthesized $32.1 \mathrm{GHz}(9.3 \mu \mathrm{m})$ (third harmonic of $28 \mu \mathrm{m}$ is $9.3 \mu \mathrm{m})$, relative to a self-locked $\mathrm{CO}_{2}$ laser (out of the photo, near the photographer). Two more locked $\mathrm{CO}_{2}$ systems offset the frequency to $29.4 \mathrm{THz}(10.2 \mu \mathrm{m})$ to be multiplied by 3 to reach the $88-\mathrm{THz}(3.39-\mu \mathrm{m})$ objective. In Fig. 3, the rightmost discharge is the 3.39- $\mu \mathrm{m}$ laser that was phase-locked to the $\mathrm{CH}_{4}$-stabilized reference laser (also out of the photo, near the photographer) which was provided by Hall and Barger. In these measurements [12], the $10-\mu \mathrm{m} \mathrm{CO}_{2}$ lasers were provided by Russ Peterson, and the full measurement team was completed by Bruce Danielson, Gordon Day, and Dick Barger. The extensive review [13] of Jennings et al. covers all laser frequency measurements before 1986, and the most modern values are given recently by David Knight [14].

In a parallel effort, by 1970, Dick Barger and John Hall had set up in JILA a new kind of precision interferometer in which the mechanical separation of the Fabry-Perot reflector plates would be controlled dynamically. Three control beams, derived from a frequency-offset-locked laser at $3.39 \mu \mathrm{m}$, illuminated three auxiliary interferometers formed by the common (moveable) mirror and three additional small mirrors fixed to the endplate of the massive invar tube which formed the interferometer spacer. When the frequency beat with the CH4-stabilized reference laser was changed by the frequency control system, a different wavelength was delivered to the auxiliary interferometer whose three positioning PZT transducers then dutifully changed the physical length by the programmed amount. The wavelength standard from 1960 to 1983 was provided by an ${ }^{86} \mathrm{Kr}$ discharge operated at the triple point of Nitrogen $\sim 63.2 \mathrm{~K}$. Because of the limited coherence length $(\sim 25 \mathrm{~cm})$ of this $605.7-\mathrm{nm}$ standard wavelength, the interferometer length was limited to this approximate value or less. Several other colors were produced in the $\mathrm{Kr}$ discharge and were conventionally used, along with the "method of exact fractions," to establish the basic length of the interferometer. Preliminary wavelength observations were already available in 1970 at the time of the international meeting of specialists concerned with length, organized by the International Bureau of Weights and Measures (BIPM) and held at their facility in Sèvres, just outside of Paris. This Comité Consultatif pour la Définition du Mètre (CCDM) forms the international focus [15] for the Length Standard and Measurement topic. Two additional years were invested in running down the myriad systematic effects that plague interferometry, especially with classical incoherent light sources. Basically all the uncertainty came from the Krypton standard, as different labs had evolved different strategies to deal with its (small) asymmetry. The wavelength of the $\mathrm{CH}_{4}$-stabilized laser was determined by these measurements [16] to be $3392.231390 \mathrm{~nm}, \pm 4 \times 10^{-9}$. Of course, when the CCDM in 1973 considered wavelength values from all the national metrology laboratories, there was also some dispersion which was resolved by an equal-weighting averaging process. This moved the speed of light up by $0.6 \mathrm{~m} / \mathrm{s}$, corresponding to a wavelength shift of $2 \times 10^{-9}$, only half the nominal uncertainty zone of the $\mathrm{Kr}$ lamp-based wavelength standard system. The analogous $\mathrm{CO}_{2}$ laser speed of light results at NRC [17] and the confirming wavelength values for methane by NRC [18] and NIST-Gaithersburg [19] supported the proposed speed of light number. The CCDM in 1973 was able to recommend a value for the speed of light, with accuracy fixed by the Krypton length standard's $\pm 4 \times 10^{-9}$ uncertainty.

Now physicists could agree to take action on the dream to redefine the Meter. We knew the wavelength and frequency of a suitable laser radiation. So $c=\lambda * \nu$, and let's all go home early [20]. But the people who actually do length measurements were not so happy: lacking infrared vision was a huge handicap for them using such a system in everyday measurements. This community of metrologists held that any decent wavelength standard would be visible, and a plethora of systems were then soon developed. Most were based on saturated absorption in $\mathrm{I}_{2}$, and many laser sources were offered from HeNe red, HeNe orange at $612 \mathrm{~nm}$, HeNe yellow at $594 \mathrm{~nm}, \mathrm{HeNe}$ red at $640 \mathrm{~nm}$, and $\mathrm{Ar}^{+}$at $514.5 \mathrm{~nm}$. One delicious demonstration by Gary Hanes [21], [64] was to use doubled radiation from HeNe at $1152 \mathrm{~nm}$ to excite a yellow absorption line in $\mathrm{I}_{2}$. This was interesting because the absolute frequency measuring business had reached, with some difficulty, the frequency measurement of this line. But the MIM diode was running out of gas.

As was often the case, a good suggestion by Chebotayev [22] was all it took to break through this barrier. He proposed an audacious scheme of four-wave mixing in neon to connect the unmeasurable HeNe $633 \mathrm{~nm}$ red line with the sum of three IR frequencies which were measureable. The idea was $633 \mathrm{~nm}$ $=1.15 \mu \mathrm{m}+2.39 \mu \mathrm{m}+3.39 \mu \mathrm{m}$, with a suitable reciprocal meaning attached to the addition. This miracle process corresponds to three stepwise transitions which in sum connected be- 
tween the same pair of levels that are responsible for the HeNe red laser line. Three IR inputs to give a phase coherent red output: it was a brilliant and enabling concept. All transitions were resonant so a maximum feasible response could be expected. Of course, the free external beams of gas lasers are only a few milliwatts, so this third-order nonlinear response could be expected to be weak. It was! Soon Evenson and Wells had built more 8-m-long power lasers for the setup of Fig. 3. Changes included moving the $\mathrm{CH}_{4}$-stabilized reference laser out in the hallway behind the photographer. An additional 8-m HeNe discharge tube accepted three IR inputs and emitted a clean (weak!) red beam to be heterodyned with the iodine-stabilized $\mathrm{HeNe}$ laser provided by Howard Layer of NBS-Gaithersburg. Cliff Pollack and Don Jennings had a 2.39- $\mu$ m color center laser in the next room, sent into the "mixer" HeNe discharge through a hole in the wall. (It was 1982, before the ubiquitous optical fiber age had arrived.) This was my idea of a heroic experiment. Basically, each of the $\sim 10$ persons involved had a spectrum analyzer or counter to read and, of course, had several servo locks to monitor and reacquire.

Finally, late one night we got the magic numbers: added together they would be the frequency of the HeNe iodine-stabilized red laser, based on the methane stabilized laser which was provided by Hall and measured in absolute terms as previously described. The final data and other numbers were written down, lasers and electronics were being switched down when a little bad news showed up: somehow in the great excitement of getting to this point I had failed to switch this $\mathrm{CH}_{4}$ reference laser from "Acquire" to "Lock"! Of course, the servo was more robust with only a high gain proportional control. Maybe I just forgot. Anyway, later measurements against the JILA Telescopic Cavity Frequency Standard showed that the proportional gain was so high that only a few kilohertz offset could have come from this cause. We also had a problem with the iodine-stabilized red laser which exhibited an anomalously large power shift. I think we ended up quoting $74 \mathrm{kHz}$ uncertainty on the 473-THz frequency, or just about $1.5 \times 10^{-10}$ uncertainty. This 1983 team core consisted of D. Jennings, C. Pollack, R. Peterson, B. Drullinger, K. Evenson, J. Wells, H. Layer, and J. Hall. In addition, many NBS and JILA support people had produced special fixtures or electronics for this measurement: their support was essential and is appreciated! Let me again express our eternal thanks to the many family members who helped and endured us during this exciting time.

Our NBS/NIST frequency measurement [23] was confirmed by Layer et al. [19] and later improved by several labs. Precision 633-nm wavelength measurements, made by NRC [24] in Canada, NPL [25] in the UK, NRLM [26] in Japan, PTB [27] in Germany and the NBS [28] in Gaithersburg, could be then used to "measure" the speed of light. Similar frequency and wavelengths measurements were made by NPL [29] for a $\mathrm{CO}_{2}$ laser. The remarkable agreement of all these values proved decisive in that the CCDM meeting of 1982 was able to agree upon the redefinition of the speed of light. A number of prominent scientists were additionally consulted regarding the proper approach and wording. The result: "The Meter is the length of the path traveled by light in vacuum in 1/299792458 of a second." This new Definition of the
Meter was adopted by the General Committee on Weights and Measures (CGPM) in October 1983.

The ending of this epoch hardly left any voids: tunable lasers at many colors have arrived and are useful. Many new frequency standards, or at least stabilization schemes have been explored. One important avenue leads to frequency measurements which are significant in that they deal with fundamental atomic systems such as hydrogen and helium. Particularly notable in this regard are the continuing refinements made by T. Hänsch's group in (Stanford and) Garching and by François Biraben and Lucille Julien at Ecole Normale in Paris.

With tunable lasers, another direction is to focus on particular quantum transitions which seem to present the best prospect for the ultimate stability. Excellent results have been obtained with laser-cooled/trapped $\mathrm{Ca}$ at PTB [30] and at NIST [31]. The Sr experiments at JILA [32] have shown two-stage trapping but not yet real high resolution. This future of the highest accuracy optical standards is believed primarily to lie with the laser-cooled trapped ions as shown in pioneering work by Dehmelt [33], [65], [66] and Wineland [34]. Unfortunately the electrostatic interactions between ions are so strong it appears that one is likely to be restricted to a single ion for this kind of atomic clock. Excellent results have been achieved with $\mathrm{Hg}^{+}, \mathrm{Sr}^{+}$and $\mathrm{In}^{+}$, and $\mathrm{Yb}^{+}$is a great favorite in view of its extremely long lifetime ( $>3 \mathrm{yrs}$ ). Some unprecedented high resolution $\left(1.6 \times 10^{14}: 1\right)$ has just been obtained with $\mathrm{Hg}^{+}$by Bergquist and colleagues [35]. Excellent stabilization and frequency measurement of the $\mathrm{Sr}^{+}$transition at $674 \mathrm{~nm}$ has recently been reported by Madej and colleagues [36]. Ekkehard Peik et al. at MPQ (Garching) have measured [37] the frequency of the $\mathrm{In}^{+}$line. Gill's group at NPL has promising early results $[38]$ on $\mathrm{Yb}^{+}$. Tamm at PTB also has $\mathrm{Yb}^{+}$resonance signals [39]. So there surely will be optical clocks with performance expected to be reaching toward $10^{-18}$ accuracy.

How will we ever conveniently be able to compare these frequencies?

As far as I know, Hänsch and Chebotayev were the first to see the dream to use the time-repetitive pulse train to form an optical frequency comb of frequencies. Hansch's work [40], [67] measured some hfs intervals in $\mathrm{Na}$ with the repetition-rate comb of a mode-locked laser. Independently, Chebotayev (Inst. of Laser Phys., Acad. of Sci., Novosibirsk, Russia) also saw the possibilities of using a repetitive pulse train for measurement [41]. He was still thinking about equivalent ideas when he visited JILA in 1988, and we talked a lot about whether one could ever get bright spectral lines with pulsed laser sources. I expected that amplitude noise would lead to random intensity-dependent phase shifts. Then one would one only have phase-noise fuzz at such a high harmonic $\left(\sim 10^{6}\right)$ of the repetition rate. Luckily, I was wrong! (see below). By the early 1990s, Chebotayev's group had a full proposed microwave/optical interconnection system and optically based atomic clock planned [42]. His untimely death in 1992 deprived us of an inexhaustible source of good physics ideas.

Next was the optical comb generator of Kourogi (Tokyo Institute of Technology) who used an efficient 6-GHz electrooptic modulator inside an optical cavity to repeatedly shift the frequency of an input optical beam, generating a few terahertz 


\section{Measuring Spectral $\delta$-functions with Temporal $\delta$-functions?!}
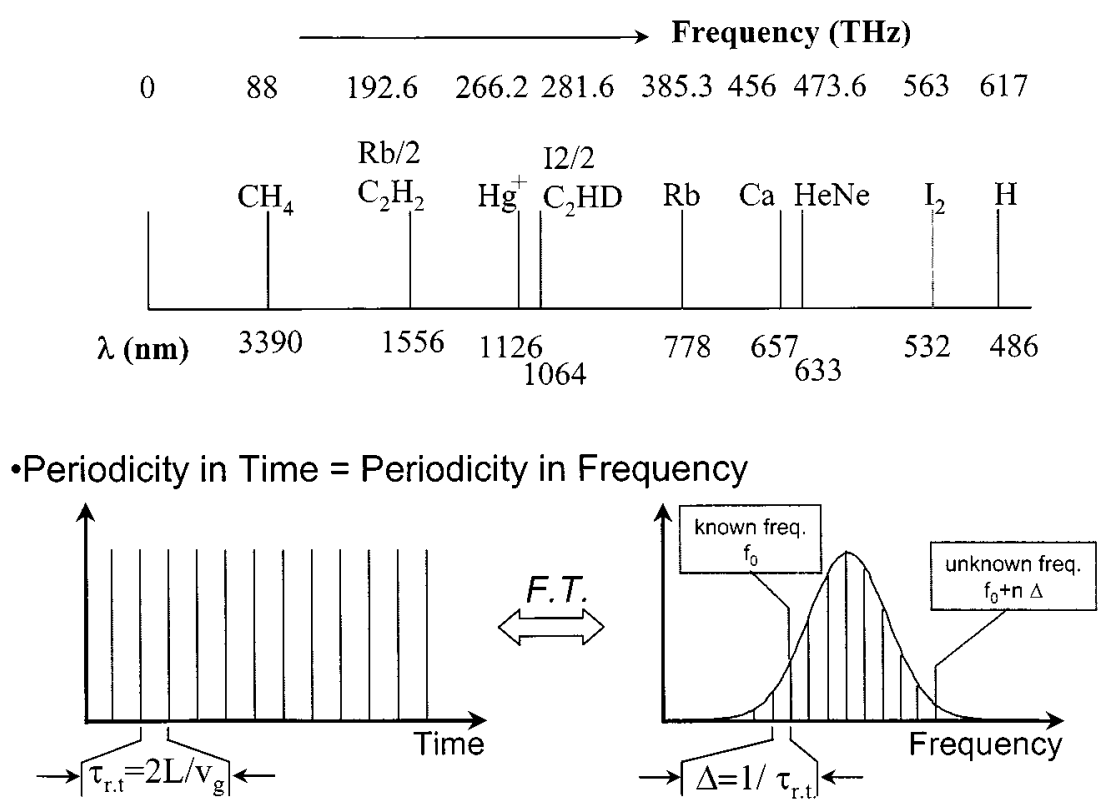

Fig. 4. Selection of stable lasers, showing approximate equality in their $\sim 90 \mathrm{THz}$ frequency spacing. In this plan, the residual "little" $\sim 20-\mathrm{THz}$ frequency intervals can be measured by a spectral comb generated by either fs laser or modulator techniques.

bandwidth, with a 6-GHz comb spacing [43]. Kourogi's comb is an important approach, especially with amplification with a 1500-nm Er-doped fiber amplifier to near watt levels of average power, which allows subsequent broadening to $50-\mathrm{THz}$ bandwidth using four-wave mixing in a dispersion-shifted fiber [44].

Even without the additional broadening, Kourogi-san's method offers a fully general way to span a few terahertz frequency interval. This is of great interest to the metrology community, as can be inferred from Fig. 4, which shows a number of the well-studied lasers' frequencies. One is struck by the approximate uniformity of the $\sim 90-\mathrm{THz}$ gaps between these frequencies: some comb-based mixing and frequency-differencing steps could almost surely provide phase-coherent connections between most of these standards. A JILA effort to increase the comb bandwidth used optical parametric amplification inside the comb-generating cavity, leading to a comb-OPO system [45]. So this avenue looked most promising. Of course, the fs $\mathrm{Ti}$ : sapphire laser offered an even broader comb width.

In the same time frame, vigorous work at Hänsch's lab (now in Garching at the Max Planck Institute for Quantum Optics) was focused on building a full frequency-measuring chain for the Hydrogen 1S-2S transition, based on the Hänsch-Telle frequency divider stage [46]. This divider system coherently divides optical frequency intervals into two equal parts. Compared with the old-time chains, where the frequencies themselves were related by the factor of two multiple in each step, in this new chain it was the frequency intervals which were so related. This was wonderful, as most all of the divider chain's lasers and mixing crystals were now basically the same, only detuned a few nanometers from each other to generate the different intervals. (A related system was built using the many lines of the $\mathrm{CO}_{2}$ lasers at 10.6 microns, by Ken Baird, Brad Whitford, and their colleagues of the NRC in Canada, and is still in use! [36]) A number of related ideas in the difference frequency synthesis scheme were proposed in about the same time frame, in the early and mid 1990s, the most notable ones include interval bisection [46], optical parametric oscillators [47], comb generation, frequency division, and four-wave mixing in diodes.

A Kourogi comb was used in Garching to span the two ends of the finest frequency interval, thus giving the interval in terms of the comb spacing, as we discussed. (The various optical intervals were not simply binary multiples, as the purpose of this whole effort was to measure the frequency of the hydrogen transition [48].) The Kourogi comb was also used to test the accuracy of the femtosecond comb [49].

Then an important simplification was made by Hänsch's Max Planck group in Garching, effectively replacing the comb generator with a femtosecond laser comb. The interval was based on the $\mathrm{CH}_{4}$-stabilized reference laser, whereby half of its $88 \mathrm{THz}$ was spanned by the comb emitted by a fs laser, enabling its absolute measurement, and then measurement of the $\mathrm{Cs}_{\mathrm{D}} \mathrm{D}_{1}$ line frequency [50]. Now far fewer divider stages were required using the femtosecond laser-based comb generator [51]. The Garching team was the first to find practical ways to independently adjust the frequency position of the comb lines and also to adjust their spacing using feedback methods [52]. This enabled precision measurement of the hydrogen frequency [53], and recently an improved measurement was referenced to a Cs Fountain Clock [54]. This represents the most accurate optical frequency measurement to date, $\pm 1.8 \times 10^{-14}$. 


\section{Direct RF to Optical Frequency Measurements}

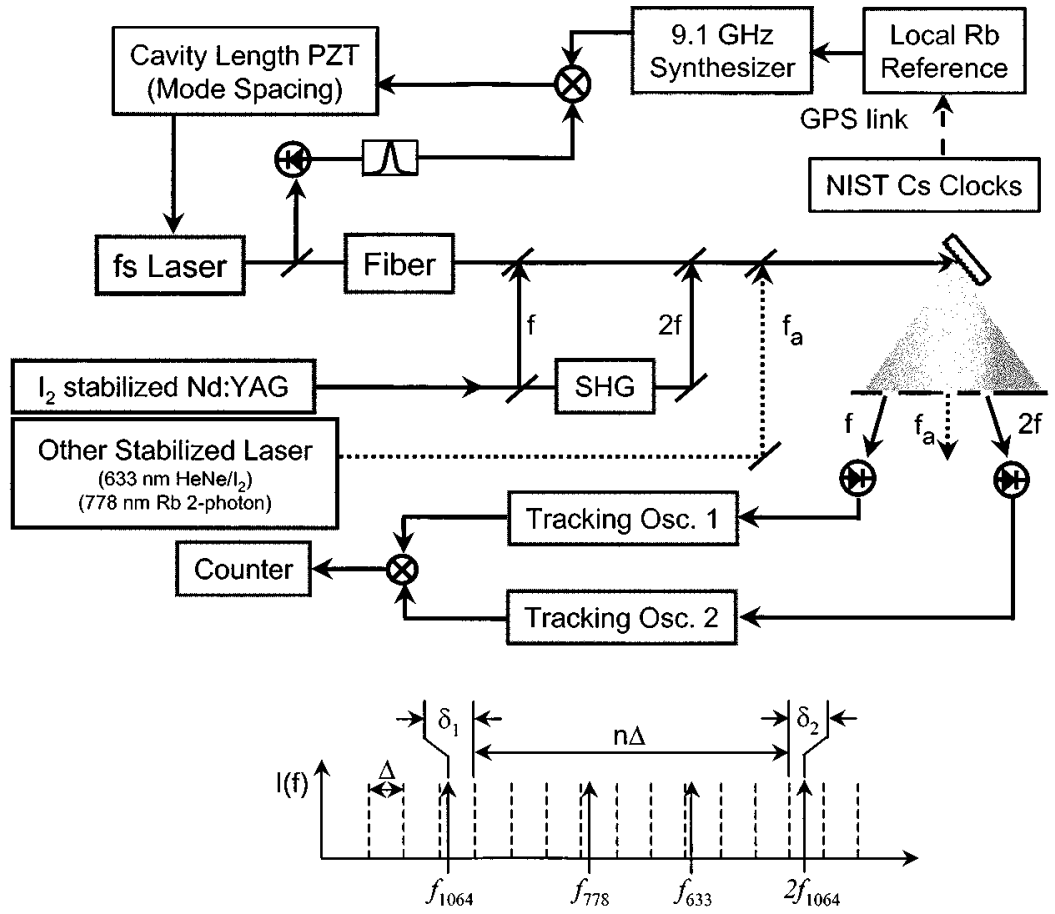

Fig. 5. Direct rf to optical frequency measurement. Stable laser is frequency-doubled from $f$ to $2 f$ and mixed with the octave-spanning comb. Heterodyne beats are obtained with components at $f$ and $2 f$, using tracking filters. With the comb now calibrated and stabilized, an unknown laser can be easily measured in terms of the rf standard.

Of course, other players became interested in Hansch's fs approach and, in early 2000 we published a paper [55] measuring across a 104-THz gap from 1064 to $778 \mathrm{~nm}$. Finally, my JILA/NIST colleague Steve Cundiff was able to get a fiber sample from the Lucent Bell Labs team of Jinendra Ranka, Robert Windeler, and Andy Stentz. Just as they had presented [56] in 1999, we instantly had octave-bandwidth fs combs. Their name "Rainbow fiber" was quite appropriate. Also, it is called "Microstructure fiber," photonic crystal fiber, and "holey" fiber." Since the JILA labs had long been in the business of stabilizing lasers, we were already prepared with well-evolved electronics for this task. However, now the reward was immense: as in the Garching experiment, we stabilized the pulse repetition rate and the frequency position of one of the comb lines. But our spectrum spanned about $400 \mathrm{THz}$ at a center frequency of $400 \mathrm{THz}$. A new stable laser, $\sim 1 \mathrm{kHz}$ wide, every $100000 \mathrm{MHz}$. That's 4 million stable lasers!

In a recent PRL [57], we report the fruition of Hänsch's vision of measuring the optical frequency directly. This can be done with the aid of our super-stable Nd : YAG laser, stabilized by locking its second harmonic to an attractive iodine line at 532 $\mathrm{nm}$. As explained in the letter and illustrated in Fig. 5, we measured the beat frequency of the Nd: YAG output with the nearest of the fs laser's comb lines. The doubled green light from the reference laser was similarly measured. Now we have the optical frequency $f$ (the frequency of the stable 1064-nm laser), which is realized as the frequency interval between $f$ and $2 f$ (the frequency of the doubled 1064-nm laser). It is expressed

\footnotetext{
${ }^{1}$ Recently, similar fibers have been fabricated by P. St. J. Russell, J. C. Knight, and colleagues at University of Bath, U.K.
}

in the lab as a large integer times the $100-\mathrm{MHz}$ repetition rate, plus or minus some differences at the ends, as noted before. So now every comb line is known with an accuracy limited only by the accuracy/stability of our house rf frequency standard. In these experiments, this rf standard was a Rb-based atomic clock, which was constantly monitored against the primary Cs clock located on the NIST campus about $1 \mathrm{~km}$ away, by means of common-view GPS measurements at both sites [58]. Recently, an optical fiber was installed so we will have good access to the NIST clock, while NIST colleagues will be able to enjoy green light from our stabilized Nd: YAG reference laser for their experiments.

The next step in this evolution was reported [59] recently and basically uses frequency doubling of some of the red comb lines to compare with fiber-generated comb lines at their doubled frequency in the blue-green. In this way, one eliminates the need for even the one extra laser. Here is now a frequency synthesis/measurement tool supreme: a single fs laser and Rainbow fiber team which emits 4 million stable laser lines, exactly $100 \mathrm{MHz}$ apart, and exactly on the $100-\mathrm{MHz}$ harmonic milestone markers, free of any possible offsets (such as shown by the laser stabilization approach discussed in this PRL). The accuracy of this approach to optical frequency synthesis has recently been confirmed at the $5 \times 10^{-16}$ level by the Garching team [60]. Another test of the mutual consistency of the measurements was made via an international intercomparison using a transportable $\mathrm{HeNe} / \mathrm{Iodine}$ laser [61].

To briefly recap a quarter century, while a NIST team (it was NBS then) measured the frequency of a stable infrared laser for the first time, another NBS effort measured its wavelength in terms of the then-standard Krypton discharge lamp, thus accu- 
rately measuring the speed of light in 1973 and setting the stage for a redefinition of the Meter. This last step was taken 10 years later by the General Committee on Weights and Measures, to give length in terms of the frequency of a laser source and a "dimensional conversion constant" $c=299792458 \mathrm{~m} / \mathrm{s}$. Getting to this conclusion absorbed the full power of $\sim 10 \mathrm{NBS}$ scientists for most of this full intervening decade, to obtain one single optical frequency measurement at an accuracy $<2 \times 10^{-10}$. Teams at the PTB in Germany and the NRC in Canada have recently pushed these traditional frequency chains to very high accuracies, just a few hundred hertz out of an optical frequency of about $400 \mathrm{THz}$, a fractional accuracy in the low $10^{-13}$ range!

Fifteen years later, more or less, the femtosecond laser spectral comb has arrived. It is characteristic of the pace of this field at present that the femtosecond results are coming in so fast that new methods are perfected before the last results appear in print. The field truly is flying at Internet speed! With the techniques evolved by this informal world-wide fs laser collaboration, we can now make optical frequency measurements in just a few minutes, and with orders of magnitude higher accuracy! The limit is not the comb method or technology: it is rather the laboratory realization of what one means by the unit of frequency, the Hertz! It is correct to view this new optical-to-microwave connectability as a revolution, a paradigm shift. In the nearest future we will see microwave frequencies of unprecedented stability being derived from stable laser sources. In five years, I expect we will have fiber-coupled optical frequency meters almost as convenient as today's GPIB-connected rf frequency counters.

Of course, events seem different to individual observers: I hope my friends and colleagues whose work has been slighted, misrepresented, or omitted will forgive me for the errors of recollection, and instead take joy from our world-wide collective achievement of making the frequency of light a directly measurable quantity.

\section{ACKNOWLEDGMENT}

The author wishes to express his appreciation to P. L. Bender for his generosity and vision in the early stages of this long project. It is a great pleasure to thank the NBS/NIST colleagues D. Jennings, K. Evenson, and J. Wells for continuing useful interactions. The NIST frequency team has expanded to include J. Bergquist, L. Hollberg, S. Diddams, C. Oates, K. Vogel, and J. Kitching. At JILA we have the stimulation of interactions with J. Ye, S. Cundiff, M. Murnane, and H. Kapteyn. The duration of this project has been so extreme that the community has lost F. R. Peterson (1983), R. L. Barger (1996), and V. Chebotayev (1992) during this time. The author is pleased to acknowledge and thank J. Ye for his laboratory finesse and his insightful comments on this manuscript. Most especially I would like to thank my dear wife Lindy for her remarkable tolerance of late nights and missed dinners, delayed or cancelled vacations, and strong general support for these 42 years.

\section{REFERENCES}

[1] K. M. Evenson, J. S. Wells, F. R. Petersen, B. L. Danielson, G. W. Day, R. L. Barger, and J. L. Hall, "Speed of light from direct frequency and wavelength measurements of the methane-stabilized laser," Phys. Rev. Lett., vol. 29, pp. 1346-1349, 1972.
[2] W. R. Bennett, Jr. and J. W. Knutson, Jr., "Simultaneous laser action on the neon doublet at $1.1523 \mu \mathrm{m}$," Proc. IEEE, vol. 52, pp. 861-862, 1964.

[3] J. L. Hall and W. W. Morey, "Optical heterodyne measurement of neon laser's millimeter wave difference frequency," App. Phys. Lett., vol. 10, pp. 152-155, 1967.

[4] K. D. Froome, "A new determination of the free space velocity of electromagnetic waves," Proc. Roy. Soc. Lond., vol. A247, pp. 109-122, 1958.

[5] P. H. Lee and M. L. Skolnick, "Saturated neon absorption inside a 6328 A laser," Appl. Phys. Lett., vol. 10, pp. 303-305, 1967.

[6] R. L. Barger and J. L. Hall, "Pressure shift and pressure broadening of methane line at $3.39 \mu \mathrm{m}$ studied by laser-saturated molecular absorption," Phys. Rev. Lett., vol. 22, pp. 4-8, 1969.

[7] J. Levine and J. L. Hall, "Design and operation of a methane absorption stabilized laser strainmeter," J. Geophys. Res., vol. 77, p. 2595, 1972.

[8] O. Acef, F. Michaud, and G. D. Rovera, "Accurate determination of $\mathrm{OsO}_{4}$ absolute frequency grid at 28/29 THz," IEEE Trans. Instrum. Meas., vol. 48, pp. 567-570, 1999. and references therein.

[9] V. P. Chebotayev, Radio Tech. -I Electron., vol. 11, p. 1712, 1966.

[10] L. O. Hocker, A. Javan, D. R. Rao, L. Frenkel, and T. Sullivan, "Absolute frequency measurement and spectroscopy of gas laser transitions in the far IR,” Appl. Phys. Lett., vol. 10, pp. 147-149, 1967.

[11] K. M. Evenson, J. S. Wells, L. M. Matarrese, and L. B. Elwell, "Absolute frequency measurements of the 28 and $78 \mu \mathrm{m}$ water vapor lines," Appl. Phys. Lett., vol. 16, pp. 159-162, 1970.

[12] K. M. Evenson, J. S. Wells, F. R. Petersen, B. L. Danielson, and G. W. Day, "Accurate frequencies of molecular transitions used in laser stabilization: The $3.39 \mu \mathrm{m}$ transition in $\mathrm{CH}_{4}$ and the 9.33 - and $10.18-\mu \mathrm{m}$ transitions in $\mathrm{CO}_{2}$," Appl. Phys. Lett., vol. 22, p. 192, 1973.

[13] D. A. Jennings, K. M. Evenson, and D. J. Knight, "Optical frequency measurements," Proc. IEEE, vol. 74, pp. 168-179, 1986.

[14] D. J. E. Knight, "Present frequency standards for lasers," in Proc. Eur. Frequency and Time Forum, Neuchatel, Switzerland, March 4-6, 1997, pp. 623-627.

[15] The name is now CCL, Consultative Committee for Length.

[16] R. L. Barger and J. L. Hall, "Wavelength of the $3.39 \mu \mathrm{m}$ laser-saturated absorption line of methane," Appl. Phys. Lett., vol. 22, pp. 196-199, 1973.

[17] K. M. Baird, H. D. Riccius, and K. J. Siemsen, " $\mathrm{CO}_{2}$ wavelengths and the velocity of light," Opt. Comm., vol. 6, pp. 91-95, 1972.

[18] K. M. Baird, D. S. Smith, and W. E. Berger, "Wavelengths of the $\mathrm{CH}_{4}$ line at $3.39 \mu \mathrm{m}$," Opt. Comm., vol. 7, pp. 107-109, 1973.

[19] H. P. Layer, R. D. Deslattes, and W. G. Schweitzer Jr., "Laser wavelength intercomparison by high resolution interferometry," Appl. Opt., vol. 15, pp. 734-743, 1976

[20] J. L. Hall, A summary of this epoch appears in, "Stabilized lasers and the speed of light," in Atomic Masses and Fundamental Constants, Sanders and Wapstra, Eds. New York: Plenum, 1976, vol. 5, pp. 322-329.

[21] G. R. Hanes, "Doubly resonant intracavity generation of second harmonic of $1153 \mathrm{~nm}$ radiation," Appl. Opt., vol. 18, p. 3970, 1979.

[22] V. P. Chebotayev, V. M. Klementyev, and Y. A. Matyugin, "Frequencysynthesis of $0.63 \mu \mathrm{m}$ radiation by mixing of 3 IR radiation frequencies in a gas," Appl. Phys., vol. 11, p. 163, 1976.

[23] D. A. Jennings, C. R. Pollock, F. R. Petersen, R. E. Drullinger, K. M. Evenson, J. S. Wells, J. L. Hall, and H. P. Layer, "Direct frequency measurement of the $\mathrm{I}_{2}$-stabilized $\mathrm{He}-\mathrm{Ne} 473-\mathrm{THz}$ (633-nm) laser," Opt. Lett., vol. 8, p. 136, 1983.

[24] G. R. Hanes, K. M. Baird, and J. DeRemigis, "Stability, reproducibility, and absolute wavelength of a $633 \mathrm{~nm} \mathrm{He}-\mathrm{Ne}$ laser stabilized to an iodine hyperfine component," Appl. Opt., vol. 12, pp. 1600-1605, 1973.

[25] W. R. C. Rowley and A. J. Wallard, "Wavelength values of the $633 \mathrm{~nm}$ laser, stabilized with ${ }^{127} \mathrm{I}_{2}$ saturated absorption," J. Physics E: Sci. Instrum., vol. 6, pp. 647-652, 1973.

[26] N. Ito and K. Tanaka, "Absolute wavelength measurements of the iodine 127 stabilized He-Ne laser of NRLM," Metrologia, vol. 14, pp. 47-51, 1978.

[27] J. Helmcke and F. Bayer-Helms, "Stabilization of an ${ }^{3} \mathrm{He}-22 \mathrm{Ne}$ laser through saturated absorption in ${ }^{129} \mathrm{I}_{2}$, " Metrologia, vol. 10, pp. 69-71, 1974.

[28] W. G. Schweitzer, E. G. Kessler Jr., R. D. Deslattes, H. P. Layer, and J. R. Whetstone, "Description, performance and wavelenghts of iodine stabilized lasers," Appl. Opt., vol. 12, pp. 2927-2938, 1973.

[29] T. G. Blaney, C. C. Bradley, G. J. Edwards, B. W. Jolliffe, D. J. E. Knight, W. R. C. Rowley, K. C. Shotton, and P. T. Woods, "Measurement of the speed of light," Nature, vol. 251, p. 46, 1976. 
[30] H. Schnatz, B. Lippart, J. Helmcke, F. Riehle, and G. Zinner, "First phase-coherent frequency measurement of visible radiation," Phys. Rev. Lett., vol. 76, p. 18, 1996.

[31] C. W. Oates, E. A. Curtis, and L. Hollberg, "Improved short-term stability of optical frequency standards: Approaching $1 \mathrm{~Hz}$ in $1 \mathrm{~s}$ with the Ca standard at $657 \mathrm{~nm}, "$ Opt. Lett., vol. 25, pp. 1603-1605, 2000.

[32] K. R. Vogel, T. P. Dinneen, A. Gallagher, and J. L. Hall, "Narrow-line Doppler cooling of strontium to the recoil limit," IEEE Trans. Instrum. Meas., vol. 48, pp. 618-621, 1999.

[33] H. G. Dehmelt, "Proposed $10^{14} \Delta \nu<\nu$ laser fluorescence on $\mathrm{T}^{+}$ mono-ion oscillator," Bull. Am. Phys. Soc., vol. 18, p. 1521, 1973.

[34] J. C. Bergquist, W. M. Itano, and D. J. Wineland, "Recoilless optical absorption and Doppler sidebands of a single ion," Phys. Rev. A, vol. A36, p. 428, 1987.

[35] R. J. Rafac, B. C. Young, J. A. Beall, W. M. Itano, D. J. Wineland, and J. C. Bergquist, "Sub-dekaHertz ultraviolet spectroscopy of ${ }^{199} \mathrm{Hg}^{+}$," Phys. Rev. Lett., vol. 85, p. 2462, 2000.

[36] J. E. Bernard, A. A. Madej, L. Marmet, B. G. Whitford, K. M. Siemsen, and S. Cundy, "Cs-based frequency measurement of a single, trapped ion transition in the visible region of the spectrum," Phys. Rev. Lett., vol. 82, p. 3228, 1999.

[37] J. von Zanthier, J. Abel, T. Becker, M. Fries, E. Peik, H. Walther, R. Holzwarth, J. Reichert, T. Udem, T. W. Hansch, A. Y. Nevsky, M. N. Skvortsov, and S. N. Bagayev, "Absolute frequency of the ${ }^{115} \mathrm{In}^{+} 5 \mathrm{~s}^{2}$ ${ }^{1} \mathrm{~S}_{0}-5 \mathrm{~s} 5 \mathrm{p}{ }^{3} \mathrm{P}_{0}$ transition," Opt. Comm., vol. 166, pp. 57-63, 1999.

[38] G. P. Barwood, P. Gill, G. Huang, H. A. Klein, and W. R. C. Rowley, "Sub-kHz 'clock' transition linewidths in a cold trapped ${ }^{88} \mathrm{Sr}^{+}$ion in low magnetic fields using 1092-nm polarization switching," Opt. Comm., vol. 151, pp. 50-55, 1998.

[39] C. Tamm, D. Engelke, and V. Bühner, "Spectroscopy of the electricquadrupole transition ${ }^{2} \mathrm{~S}_{1 / 2}(F=0)_{-2} \mathrm{D}_{3 / 2}(F=2)$ in trapped ${ }^{171} \mathrm{Yb}^{+}, "$ Phys. Rev. A, vol. A61, 2000. 053405(9).

[40] A. I. Ferguson, J. N. Eckstein, and T. W. Hänsch, "High resolution twophoton spectroscopy with picosecond light pulses," Phys. Rev. Lett., vol. 40, pp. 847-850, 1978.

[41] Y. V. Baklanov and V. P. Chebotayev, "Narrow resonances of 2-photon absorption of super-narrow pulses in a gas," Appl. Phys., vol. 12, pp. 97-99, 1977.

[42] S. N. Bagayev, V. P. Chebotayev, and V. M. Klementyev, "Time Fourier superhigh resolution spectroscopy," Laser Physics, vol. 4, pp. 293-296, March-April 1994.

[43] M. Kourogi, K. Nakagawa, and M. Ohtsu, "Wide-span optical frequency comb generator for accurate optical frequency difference measurements," IEEE J. Quantum Electron., vol. 29, p. 2692, 1993.

[44] K. Imai, M. Kourogi, and M. Ohtsu, " $30 \mathrm{THz}$ span optical frequency comb generation by self-phase modulation in an optical fiber," IEEE J. Quantum Electron., vol. 34, pp. 54-60, 1998.

[45] S. A. Diddams, L.-S. Ma, J. Ye, and J. L. Hall, "Broadband optical frequency comb generation with a phase-modulated parametric oscillator," Opt. Lett., vol. 24, p. 1749, 1999.

[46] H. R. Telle, D. Meschede, and T. W. Hänsch, "Realization of a new concept for visible frequency division: Phase-locking of harmonic and sum frequencies," Opt. Lett., vol. 15, p. 532, 1990.

[47] N. C. Wong, "Optical to microwave frequency chain utilizing a two laser based OPO network," Appl. Phys. B, vol. 61, p. 1439, 1995.

[48] T. Udem, A. Huber, B. Gross, J. Reichert, M. Prevedelli, M. Weitz, and T. W. Hänsch, "Phase-coherent measurement of the hydrogen 1S-2S transition frequency with an optical frequency interval divider chain," Phys. Rev. Lett., vol. 79, pp. 2646-2649, 1997.

[49] T. Udem, J. Reichert, T. W. Hansch, and M. Kourogi, "Accuracy of optical frequency comb generators and optical frequency interval divider chains," Opt. Lett., vol. 23, pp. 1387-1389, 1998.

[50] T. Udem, J. Reichert, R. Holzwarth, and T. W. Hänsch, "Absolute optical frequency measurement of the cesium D1 line with a mode-locked laser," Phys. Rev. Lett., vol. 82, p. 3568, 1999.

[51] _ " "Accurate measurement of large optical frequency differences with a mode-locked laser," Opt. Lett., vol. 24, p. 881, 1999.

[52] J. Reichert, R. Holzwarth, T. Udem, and T. W. Hänsch, "Measuring the frequency of light with mode-locked lasers," Opt. Comm., vol. 172, pp. $59-68,1999$.
[53] J. Reichert, M. Niering, R. Holzwarth, M. Weitz, T. Udem, and T. W. Hänsch, "Phase coherent vacuum-ultraviolet to radio frequency comparison with a mode-locked laser," Phys. Rev. Lett., vol. 84, p. 3232, 1999.

[54] M. Niering, R. Holzwarth, J. Reichert, P. Pokasov, T. Udem, M. Weitz, T. W. Hänsch, P. Lemonde, G. Santarelli, M. Abgrall, P. Laurent, C. Salomon, and A. Clairon, "Measurement of the hydrogen 1S-2S transition frequency by phase coherent comparison with a microwave cesium fountain clock," Phys. Rev. Lett., vol. 84, p. 5496, 2000.

[55] S. A. Diddams, D. J. Jones, L.-S. Ma, S. T. Cundiff, and J. L. Hall, “Optical frequency measurement across a $104 \mathrm{THz}$ gap with a femtosecond laser frequency comb," Opt. Lett., vol. 25, p. 186, 2000.

[56] J. Ranka, R. Windeler, and A. Stentz, "Visible continuum generation in air-silica microstructure optical fibers with anomalous dispersion at 800 nm," Opt. Lett., vol. 25, p. 25, 2000.

[57] S. A. Diddams, D. J. Jones, J. Ye, S. T. Cundiff, and J. L. Hall, "Direct link between microwave and optical frequencies with a $300 \mathrm{THz}$ optical comb," Phys. Rev. Lett., vol. 84, pp. 5102-5105, May 2000.

[58] L. Nelson, J. Levine, T. Parker, K. Larson, P. Hetzel, and J. Becker, "Comparison of atomic frequency standards at NIST and PTB using carrier-phase GPS," in 31st Annual Precise Time and Time Interval (PTTI) Meeting, (L. A. Breakiron, Ed: U.S. Naval Observatory, 2000), pp. 449-459.

[59] D. J. Jones, S. A. Diddams, J. K. Ranka, A. Stentz, R. S. Windeler, J. L. Hall, and S. T. Cundiff, "Carrier-envelope phase control of femtosecond mode-locked lasers and direct optical frequency aynthesis," Science, vol. 288, pp. 635-639, Apr. 2000.

[60] R. Holzwarth, T. Udem, T. W. Hänsch, J. C. Knight, W. J. Wadsworth, and P. St. J. Russell, "Optical frequency synthesizer for precision spectroscopy," Phys. Rev. Lett., vol. 85, p. 2264, 2000.

[61] J. Ye, T. H. Yoon, A. A. Madej, J. E. Bernard, K. J. Siemsen, L. Marmet, J.-M. Chartier, A. Chartier, and J. L. Hall, "Accuracy comparison of optical frequency measurement between harmonic-generation synthesis and a frequency division femtosecond-comb," Phys. Rev. Lett., vol. 85, pp. 3797-3800, 2000.

[62] K. D. Froome and L. Essen, The Velocity of Light and Radio Waves. New York: Academic, 1969.

[63] T. G. Blaney, C. C. Bradley, G. J. Edwards, and D. J. Knight, "Absolute frequency measurements of a Lamb-dip stabilized water vapour laser oscillating at $10.7 \mathrm{THz}(28 \mu \mathrm{m}), "$ Phys. Lett., vol. 43A, pp. 471-472, 1973.

[64] K. M. Baird, K. M. Evenson, G. R. Hanes, D. A. Jennings, and F. R. Petersen, "Extension of absolute frequency measurements to the visible: Frequencies of ten hyperfine components of iodine," Opt. Lett., vol. 4, p. $263,1979$.

[65] H. G. Dehmelt, "Proposed $10^{14} \Delta \nu<\nu$ laser fluorescence on $\mathrm{T}^{+}$ mono-ion oscillator," Bull. Am. Phys. Soc., vol. 20, p. 60, 1975.

[66] H. G. Dehmelt, "Mono-ion oscillator as potential ultimate laser frequency standard," IEEE Trans. Instrum. Meas., vol. IM-31, pp. 83-87, 1982.

[67] A. I. Ferguson, J. N. Eckstein, and T. W. Hänsch, J. Appl. Phys., vol. 49, pp. 5389-5391, 1978.

John L. Hall was born in 1934 in Denver, CO. He received the Ph.D. degree in physics from the Carnegie Institute of Technology (now Carnegie-Mellon University) in Pittsburgh, PA.

Since 1963, he has been with the JILA (formerly Joint Institute for Laboratory Astrophysics), the University of Colorado, and the National Institute of Standards and Technology (NIST), Boulder, where he has been responsible for a number of major innovations and developments in sensitive and high resolution laser spectroscopy, laser frequency stabilization, laser/atom cooling, quantum optics, and high precision measurements using stable laser technology.

Dr. Hall is a member of the National Academy of Sciences, a Senior Fellow of the National Institute of Standards and Technology, and a Fellow of JILA, the Optical Society of America, and the American Physical Society. 